\title{
Genotyping of clinical varicella-zoster virus isolates collected from Yunnan in Southwestern China
}

\author{
YUNLONG LI ${ }^{1,2}$ and BAOSHENG ZHU ${ }^{1,2}$ \\ ${ }^{1}$ Medical Faculty, Affiliated Hospital of Kunming University of Science and Technology (The First People's Hospital of \\ Yunnan Province), Kunming University of Science and Technology, Kunming, Yunnan 650500; ${ }^{2}$ Genetic Diagnosis Center, \\ Key Laboratory for Birth Defects and Genetic Diseases, The First People's Hospital of Yunnan Province, \\ Kunming, Yunnan 650032, P.R. China
}

Received June 12, 2015; Accepted July 22, 2015

DOI: $10.3892 /$ br.2015.562

\begin{abstract}
Varicella-zoster virus (VZV) belongs to the $\alpha$-herpesvirus family. Genetically, it is stable and is divided into several genotypes based upon the genetic variations. The genotypes of VZV are rarely studied in the Southwestern region of China. In the present study, the common genetic variations in the VZV genes were examined in $42 \mathrm{VZV}$ isolates collected from the patients with herpes zoster in the Yunnan province (Southwestern China). The restriction fragment length polymorphism analysis of open reading frames (ORFs) 38, 54 and 62 in the VZV genes showed that all the collected VZV isolates were PstI, BglI and SmaI positive. The R5 variable-repeat region in these isolates was variable (R5A: $46.4 \%$; R5B: $53.6 \%$ ). The sequencing data of ORFs 1, 21, 22 and 54 indicated that 41 of the 42 collected VZV isolates could be grouped into genotype J or J1. Only one VZV isolate was identified as genotype A1 or M2. No new substitutions in the sequenced fragments were found in the collected VZV isolates. The results of the present study provided a preliminary genetic characterization of the VZV strains in the Yunnan province of Southwestern China.
\end{abstract}

Correspondence to: Dr Yunlong Li, Medical Faculty, Affiliated Hospital of Kunming University of Science and Technology (The First People's Hospital of Yunnan Province), Kunming University of Science and Technology, 727 Jing Ming Nan Road, Kunming, Yunnan 650500, P.R. China

E-mail: yunlongli.km@gmail.com

Professor Baosheng Zhu, Genetic Diagnosis Center, Key Laboratory for Birth Defects and Genetic Diseases, The First People's Hospital of Yunnan Province, 157 Jinbi Road, Kunming, Yunnan 650032, P.R. China

E-mail: bszhu@aliyun.com

Key words: varicella-zoster virus, genotype, genomic variations, zoster, open reading frames

\section{Introduction}

Varicella-zoster virus (VZV) belongs to the $\alpha$-herpesvirus family $(1,2)$. VZV can infect humans and vertebrates (including chimpanzees and gorillas). The primary VZV infection in humans results in chickenpox. Following the recovery from chickenpox, the VZV becomes dormant in the sensory ganglia-like dorsal root ganglia (3,4). In certain conditions, VZV can reactivate and cause herpes zoster. There is a difference in onset time of primary VZV infection in different geographic regions (4). Generally, the infection time in people who have lived in temperate regions is earlier than those who have lived in tropical regions. In temperate regions, the first infection of VZV is mostly found in children, and in the tropical regions, the first infection is delayed until adulthood.

The genome of VZV is a linear duplex DNA molecule (5). The genome of VZV contained $\sim 25,000$ base pairs, including $\geq 70$ open reading frames (ORFs). Genetic variations have been identified in the genome of VZV strains (6-13). Based on the specific single-nucleotide polymorphisms (SNPs) in ORFs 1, 21, 50 and 54, VZV strains have been divided into the following genotypes: A (Africa/Asia), B and C (Europe and North America), and J (Japanese) (6). By analyzing a short region of nucleotides [447 base pairs (bp)] in ORF 22, VZV strains could be divided into genotype $\mathrm{E}$ (Europe), genotype isolates $\mathrm{J}$ (Japanese) and genotype M (Mosaic) (13). The genotype M strains can be further divided into genotypes of M1, M2, M3 and M4 (13-15). The R5 variable regions (R5A and R5B) between ORF60 and ORF61 are also used to distinguish the genotypes of VZV strains $(10,11)$. By phylogenetic analysis of the complete VZV genomes, the VZV strains could be broadly grouped into 5 clades (clades 1 and 3: European/North American strains; clade 2: Asian strains, particularly for the strains from Japan; clade 4: Certain strains from Europe; and clade 5: Indian strains) (16).

Until now, the VZV strains in China were not extensively studied. In the study by Loparev et al (13), 6 VZV samples in China were analyzed. The 3 samples in South China were all type M2. The other 3 samples in North China were all type E. Liu et al (17) collected 19 VZV samples from the patients with zoster or varicella in Hefei city of Anhui province, which is located in the middle Eastern part of China (18). The collected 
Table I. Primers used for amplifying the fragments in the VZV genes.

\begin{tabular}{|c|c|c|c|c|}
\hline Gene & Primer sequences $\left(5^{\prime} \rightarrow 3^{\prime}\right)$ & Size, bp & Note & (Refs.) \\
\hline ORF1 & $\begin{array}{l}\text { F: TCAGCTGGCTTTTCTAAGAATTCG } \\
\text { R: TATTTTTGGGATCCGCAATGTC }\end{array}$ & 506 & $\begin{array}{l}\text { PCR; sequencing } \\
\text { PCR; Sequencing }\end{array}$ & (9) \\
\hline ORF 21 & $\begin{array}{l}\text { F: TGGCGCGGTTTAAATGAATTGA } \\
\text { R: CACGTGTAGCTCCAAAAACCTAGG }\end{array}$ & 503 & $\begin{array}{l}\text { PCR; sequencing } \\
\text { PCR; sequencing }\end{array}$ & (9) \\
\hline ORF 22 & $\begin{array}{l}\text { F: GGGTTTTGTATGAGCGTTGG } \\
\text { R: CCCCCGAGGTTCGTAATATC }\end{array}$ & 447 & $\begin{array}{l}\text { PCR; sequencing } \\
\text { PCR; sequencing }\end{array}$ & (9) \\
\hline ORF38 & $\begin{array}{l}\text { F: AAGTTTCAGCCAACGTGCCAATAAA } \\
\text { R: AGACGCGCTTAACGGAAGTAACG }\end{array}$ & 647 & $\begin{array}{l}\text { PCR; RFLP analysis }(P s t \mathrm{I}) \\
\text { PCR; RFLP analysis }(P s t \mathrm{I})\end{array}$ & (9) \\
\hline ORF54 & $\begin{array}{l}\text { F: CGTAATGCATAACAGGCCAACAC } \\
\text { R: AAACCTGGCGTCAAACATTACA }\end{array}$ & 497 & $\begin{array}{l}\text { PCR; RFLP analysis (BgII); sequencing } \\
\text { PCR; RFLP analysis (BgII); sequencing }\end{array}$ & (9) \\
\hline$R 5$ & $\begin{array}{l}\text { F: GGCAAATACTTAGACCGTTTT } \\
\text { R: TAATGGACTTTTAATGGATTG }\end{array}$ & $\begin{array}{l}359(\mathrm{R} 5 \mathrm{~A}) \\
471(\mathrm{R} 5 \mathrm{~B})\end{array}$ & $\begin{array}{l}\text { PCR } \\
\text { PCR }\end{array}$ & (10) \\
\hline ORF62 & $\begin{array}{l}\text { F: TTCCCACCGCGGCACAAACA } \\
\text { R: GGTTGCTGGTGTTGGACGCG }\end{array}$ & 268 & $\begin{array}{l}\text { PCR; RFLP analysis }(S m a I) \\
\text { PCR; RFLP analysis }(S m a I)\end{array}$ & (12) \\
\hline
\end{tabular}

RFLP, restriction fragment length polymorphism; R5A, R5 variable region type A; R5B, R5 variable region type B; ORF, open reading frame; VZV, varicella-zoster virus; PCR, polymerase chain reaction; F, forward; R, reverse.

VZV isolates were grouped into genotype $\mathrm{J}$ or $\mathrm{J} 1$. The genotype of R5A and R5B were found in these isolates by analyzing the R5 variable region. In the present study, $42 \mathrm{VZV}$ isolates were collected from Yunnan province of Southwestern China. Using the described genotyping method of Liu et al (17), a genetic characterization of the VZV strains was made in Southwestern China.

\section{Materials and methods}

Patients and clinical samples. The VZV isolates were collected from the 42 ambulatory and hospitalized patients with herpes zoster in The First People's Hospital of Yunnan Province (The Affiliated Hospital of Kunming University of Science and Technology, Kunming, Yunnan, China) between August 2013 and December 2014. Vesicle fluid was collected from skin lesions, and the collected samples were stored at $-20^{\circ} \mathrm{C}$. The patients were all Han Chinese in the Yunnan province of Southwestern China. The study was approved by the Institutional Ethics Committee of the First People's Hospital of Yunnan Province. The written informed consent was signed and obtained from all the patients who participated. The study was performed according to the principles of the Declaration of Helsinki. Genomic DNA of VZV was extracted from vesicle fluid from skin lesions using a viral DNA extraction kit (Da An Gene Co., Ltd., of Sun Yat-Sen University, Guangzhou, China). The collected isolates were first detected by nested polymerase chain reaction (PCR) and quantitative PCR, as described by Weidmann et al (19). The results of the nested PCR and quantitative PCR showed that all the collected isolates exhibited the VZV gene.

PCR and sequencing. PCR was performed in a $25-\mu 1$ reaction mixture containing 10X LA PCR Buffer II ( $\mathrm{Mg}^{2+}$ Plus),
2.5 units of TaKaRa LA Taq (Takara Bio, Inc., Dalian, China), $50 \mu \mathrm{M}$ of each deoxyribonucleotide, $0.2 \mu \mathrm{M}$ of each primer and $50 \mathrm{ng}$ DNA. The primers for amplifying and sequencing the fragments of the VZV genes referred to the previous studies by Liu et al (17), Barrett-Muir et al (6), Hawrami and Breuer (10) and Loparev et al (13), and are shown in Table I. The conditions for PCR amplification was as follows: A denaturation cycle at $94^{\circ} \mathrm{C}$ for $5 \mathrm{~min}$, followed by 35 cycles at $94^{\circ} \mathrm{C}$ for $30 \mathrm{sec}$, annealing at $55^{\circ} \mathrm{C}$ for $30 \mathrm{sec}$ and extension at $72^{\circ} \mathrm{C}$ for $40 \mathrm{sec}$, and ending with a final extension at $72^{\circ} \mathrm{C}$ for $7 \mathrm{~min}$.

The PCR products of ORFs 1, 21, 22 and 54 in the VZV genes were purified using Genomic DNA Purification kits (Tiangen Biotech, Beijing, China) and were sequenced using the forward and reverse primers and the Big Dye Terminator version 3.1 Cycle Sequencing kit (Applied Biosystems, Carlsbad, CA, USA) on an ABI PRISM 3730 DNA sequencer (Applied Biosystems).

Restriction enzyme reactions. Restriction endonuclease digestion was performed in a $20 \mu 1$ mixture containing $7 \mu 1 \mathrm{PCR}$ products, $1 \mu \mathrm{l}$ endonuclease (BglI, PstI or SmaI; Takara Bio Inc.), $2 \mu \mathrm{l}$ of the accompanying $10 \mathrm{X}$ endonuclease buffer and $13 \mu \mathrm{l}$ sterile water. The reaction mixture for BglI and PstI was incubated at $37^{\circ} \mathrm{C}$ for $3 \mathrm{~h}$. The reaction mixture for $S m a \mathrm{I}$ was incubated at $30^{\circ} \mathrm{C}$ for $3 \mathrm{~h}$. The digested products of $B g l \mathrm{I}, P s t \mathrm{I}$ and $S m a \mathrm{I}$ were resolved by $6 \%$ polyacrylamide gels and were silver stained prior to visualization. The genotyping data were further validated by sequencing 2 randomly selected VZV samples.

\section{Results}

Restriction fragment length polymorphism (RFLP) analysis of ORF 38, 54 and 62. The specific SNPs in the VZV strains that 
Table II. Genotype of the VZV strains in the present study and other studies.

\begin{tabular}{lcccccc}
\hline VZV strain & $\begin{array}{c}\text { ORF38 } \\
(P s t)\end{array}$ & $\begin{array}{c}\text { ORF54 } \\
(\text { BglI })\end{array}$ & $\begin{array}{c}\text { ORF62 } \\
(\text { SmaI })\end{array}$ & R5 type (\%) & $\begin{array}{c}\text { SNP in } \\
\text { ORF22 }\end{array}$ & $\begin{array}{c}\text { SNP in } \\
\text { ORFs 1,21 and 54 }\end{array}$ \\
\hline MLS (17) & + & + & - & R5A & M1 & A1 \\
v-Oka (17) & - & + & + & R5B & J & J2 \\
p-Oka (17) & - & + & - & R5B & J & J1 \\
VZV isolates from & + & + & - & R5A (47.4) & J & J1 \\
Anhui city of China (18) & & & & R5B (52.6) & J (41/42) & J1 (41/42) \\
VZV isolates from & + & + & + & R5A (46.4) & M2 (1/42) & A1 (1/42) \\
Yunnan province (present study) & & & & R5B (53.6) & .
\end{tabular}

Varicella-zoster virus (VZV) genotyping scheme using single-nucleotide polymorphism (SNP) in open reading frame (ORF) 22 referred to the description by Loparev et al (13). The genotyping scheme by using SNP in ORFs 1,21 and 54 referred to the description by Barrett-Muir et al (6) and Quinlivan et al (23). R5A, R5 variable region type A; R5B, R5 variable region type B.
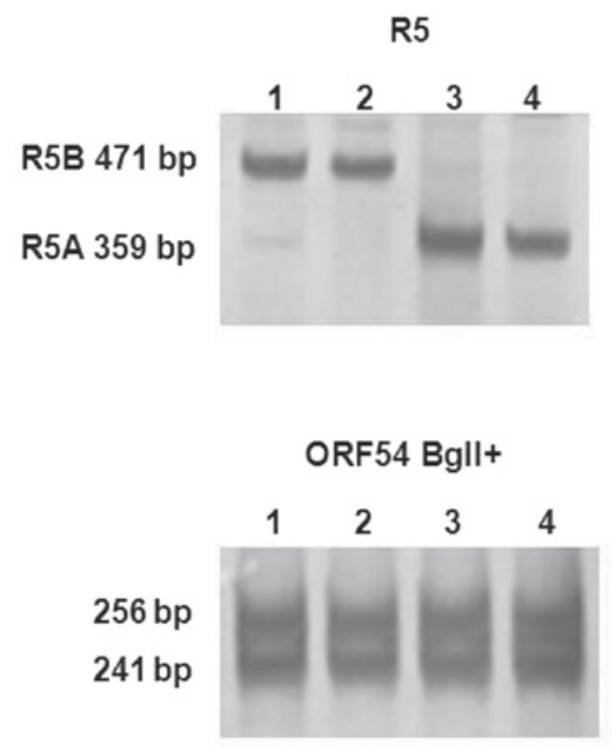

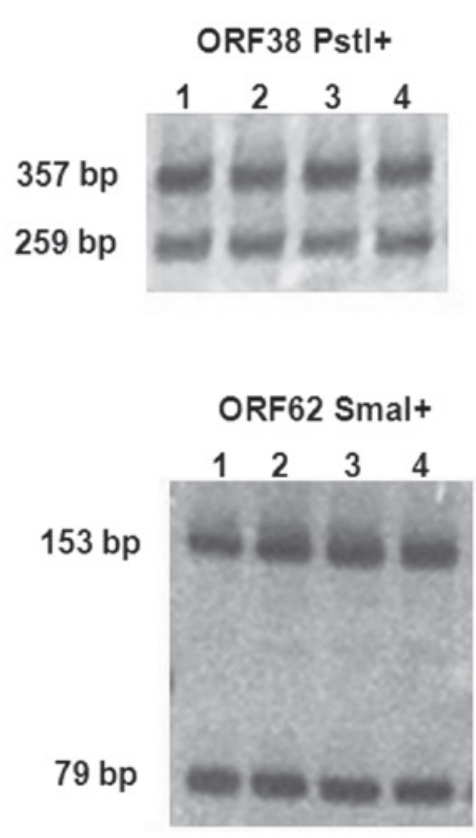

ORF38 Pstl+

ORF62 Smal+

Figure 1. Representative results of the R5 variable region and restriction fragment length polymorphism analysis of open reading frame (ORF) 38,54 and 62 .

are located in ORFs 38, 54 and 62 have been used as genetic markers in the study of VZV epidemiology. The PCR products of ORFs 38, 54 and 62 in the VZV genes were amplified and they were 647, 497 and 268 bp, respectively. RFLP analysis was used to determine the genotype of ORF38 (PstI), ORF54 $(B g l \mathrm{I})$ and ORF62 (SmaI) in the collected VZV isolates, as shown in Fig. 1. The PCR products of ORF38 were digested by PstI, and produced 2 fragments of 357 and $290 \mathrm{bp}$. The digestion of PCR products of ORF54 by $B g l$ yielded 2 fragments of 256 and $241 \mathrm{bp}$. Similarly, the PCR products of ORF62 were digested by SmaI, and produced 3 fragments of 153, 79 and $36 \mathrm{bp}$. These data indicated that the VZV isolates collected in the study contained the cleaving site of PstI, BglI and SmaI, and that the collected isolates were all $B g l \mathrm{I}$ positive $\left(B g l \mathrm{I}^{+}\right)$, Pst $\mathrm{I}^{+}$and $\operatorname{SmaI}^{+}$(Table II).

Analysis of the $R 5$ variable region. The R5 variable region has been shown to be vary among different VZV strains. The
R5 variable region was amplified by PCR and analyzed by electrophoresis to determine its distribution in the collected VZV isolates. The representative results are shown in Fig. 1. The PCR products of R5A were $359 \mathrm{bp}$, while the PCR products of R5B were $471 \mathrm{bp}$. In the collected VZV isolates, R5A and R5B were observed. The percentages of R5A and R5B were 46.4 and $53.6 \%$, respectively, in the collected isolates.

Nucleotide sequence analysis of ORFs 1, 21, 22 and 54. The sequence of ORFs 1, 21, 22 and 54 in VZV genes have been sequenced and analyzed in certain areas of the world, such as the United Kingdom, Brazil and Anhui city of China. Therefore, a nucleotide sequence analysis was performed on ORFs 1, 21, 22 and 54 in the collected VZV isolates. The informative polymorphic markers on the regions are shown in Fig. 2. Based on the genotyping scheme of Barret-Muir et al (6), the VZV isolates in the study could be grouped into genotype J1 (41/42) or A1 (1/42), as shown in 


\begin{tabular}{|c|c|c|c|c|c|c|c|c|c|c|c|c|c|c|c|c|c|c|c|c|c|c|c|c|c|c|c|c|c|}
\hline Gene & & & & & & & OR & & & & & & & & & & & & & & & & & & & & & & \\
\hline $\begin{array}{l}\text { Nucleotide } \\
\text { Position }\end{array}$ & कू & \% & \&ू & ర్ర & मु & డ్ర & ळ్ & वु & $\Phi$ & Ф్ळ & ন্ট & I্ & $\bar{్}$ & : & 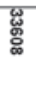 & 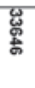 & 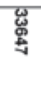 & $\underset{\underset{N}{\mathbb{N}}}{\mathbb{E}}$ & $\underset{\mathbb{N}}{\mathscr{E}}$ & $\underset{\underset{\Phi}{\Psi}}{\mathscr{W}}$ & 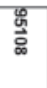 & 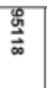 & 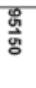 & 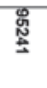 & 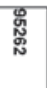 & 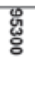 & 思 & 惫 & 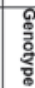 \\
\hline Dumas & $T$ & G & $G$ & $T$ & G & $T$ & A & $G$ & $T$ & $T$ & $T$ & $T$ & $T$ & c & c & $G$ & A & $T$ & $T$ & $T$ & $c$ & G & $T$ & $T$ & $G$ & c & $T$ & c & c \\
\hline MLS [17] & $\begin{array}{lll}T \\
\end{array}$ & G & 6 & $T$ & 6 & $T$ & A & 0 & $\begin{array}{ll}T \\
\end{array}$ & c & c & c & $T$ & $\mathrm{c}$ & c & G & A & c & c & c & $\mathrm{c}$ & $G$ & $T$ & c & $G$ & $T$ & $\begin{array}{ll} \\
\end{array}$ & $\mathrm{c}$ & A1 \\
\hline voka [1]] & c & $G$ & A & c & G & $c$ & A & $G$ & $\begin{array}{ll}T \\
\end{array}$ & c & c & c & $\begin{array}{ll} \\
\end{array}$ & $\mathrm{c}$ & c & G & A & c & $\mathrm{c}$ & c & $\mathrm{c}$ & G & $T$ & c & $G$ & c & $T$ & c & J2 \\
\hline poka [17] & $T$ & G & A & $T$ & G & $T$ & A & G & $T$ & c & c & c & $T$ & c & c & $G$ & A & c & c & c & $c$ & G & $T$ & $c$ & $G$ & c & $T$ & c & J1 \\
\hline 1 & $\begin{array}{lll}T \\
\end{array}$ & G & A & $T$ & G & $T$ & A & G & $\begin{array}{ll}T \\
\end{array}$ & c & c & c & $T$ & $\mathrm{c}$ & c & G & A & $c$ & $c$ & c & $\mathrm{c}$ & $G$ & $T$ & c & $G$ & c & $\begin{array}{ll} \\
\end{array}$ & $\mathrm{c}$ & J1 \\
\hline 2 & $T$ & G & A & $T$ & G & $T$ & A & 0 & $T$ & c & c & c & $T$ & $\mathrm{c}$ & c & $G$ & $A$ & c & $\mathrm{c}$ & c & $c$ & $G$ & $T$ & c & $G$ & c & $T$ & c & \begin{tabular}{|l} 
J1 \\
\end{tabular} \\
\hline 3 & $T$ & G & A & $T$ & G & $\mathrm{T}$ & A & G & $T$ & c & c & c & $T$ & c & c & G & A & c & c & c & $\mathrm{c}$ & G & $T$ & c & $\mathrm{G}$ & c & $T$ & c & J1 \\
\hline 4 & $\begin{array}{lll}T \\
\end{array}$ & G & A & $T$ & G & $T$ & A & G & $\begin{array}{lll}T \\
\end{array}$ & c & c & c & $\begin{array}{ll} \\
\end{array}$ & $\mathrm{c}$ & c & G & A & c & c & c & $\mathrm{c}$ & G & $\begin{array}{lll} \\
\end{array}$ & c & $G$ & c & $\begin{array}{lll}T \\
\end{array}$ & c & $\mathrm{J1}$ \\
\hline 5 & $T$ & $G$ & A & $T$ & $G$ & $T$ & A & 0 & $T$ & c & c & c & $T$ & c & c & $G$ & A & $c$ & c & c & $c$ & $G$ & $T$ & $\mathrm{c}$ & $G$ & c & $T$ & c & J1 \\
\hline 6 & $T$ & $G$ & A & $T$ & $G$ & $T$ & A & $G$ & $T$ & c & C & c & $T$ & c & c & $G$ & A & c & c & c & $\mathrm{c}$ & $G$ & $T$ & C & $G$ & c & $T$ & c & J1 \\
\hline 7 & $\begin{array}{lll} \\
\end{array}$ & G & A & $\begin{array}{lll}T \\
\end{array}$ & G & $T$ & A & G & $\begin{array}{lll} \\
\end{array}$ & c & c & c & $T$ & c & c & G & A & c & c & c & $c$ & G & $T$ & $\mathrm{c}$ & G & c & $T$ & c & J1 \\
\hline 8 & $T$ & $G$ & A & $T$ & G & $T$ & A & $G$ & $T$ & c & C & c & $T$ & $\mathrm{C}$ & $\mathrm{c}$ & $G$ & $A$ & $c$ & c & c & $\mathrm{c}$ & $G$ & $T$ & c & $G$ & c & $T$ & c & J1 \\
\hline 9 & $T$ & G & A & $T$ & $G$ & $T$ & $A$ & 0 & $T$ & c & C & C & $T$ & C & c & $G$ & $A$ & $c$ & C & c & c & $G$ & $T$ & c & $G$ & c & $T$ & c & J1 \\
\hline 10 & $T$ & G & G & $T$ & G & $\mathrm{T}$ & A & G & $T$ & $\mathrm{c}$ & $\mathrm{c}$ & C & $T$ & $\mathrm{C}$ & $\mathrm{c}$ & G & $A$ & $\begin{array}{ll}\mathrm{c} \\
\end{array}$ & $\mathrm{c}$ & c & $\mathrm{c}$ & G & $\mathrm{T}$ & $\mathrm{c}$ & G & $\mathrm{c}$ & $T$ & $\mathrm{c}$ & A1 \\
\hline 11 & $T$ & G & A & $T$ & G & $T$ & A & G & $T$ & c & c & c & $T$ & c & $c$ & $G$ & $A$ & $c$ & c & c & $\mathrm{c}$ & G & $T$ & c & $G$ & c & $T$ & c & J1 \\
\hline 12 & $T$ & $G$ & A & $T$ & $G$ & $T$ & A & $G$ & $T$ & $\mathrm{c}$ & $\mathrm{c}$ & $c$ & $T$ & $\mathrm{c}$ & $\mathrm{c}$ & $G$ & $A$ & $\mathrm{c}$ & $\mathrm{c}$ & $\mathrm{c}$ & $\mathrm{c}$ & $G$ & $T$ & $\mathrm{c}$ & $G$ & $\mathrm{c}$ & $T$ & $\mathrm{c}$ & J1 \\
\hline 13 & $T$ & $G$ & A & $T$ & $G$ & $T$ & A & $G$ & $T$ & $\mathrm{C}$ & C & c & $T$ & $\mathrm{C}$ & $\mathrm{c}$ & $G$ & $A$ & c & $\mathrm{C}$ & $\mathrm{c}$ & $\mathrm{c}$ & $G$ & $T$ & $\mathrm{c}$ & $G$ & $\mathrm{c}$ & $T$ & $\mathrm{c}$ & J1 \\
\hline 14 & $T$ & G & A & $T$ & G & $T$ & A & G & $T$ & c & c & $c$ & $T$ & $\mathrm{c}$ & $\mathrm{c}$ & G & A & $c$ & c & c & $\mathrm{c}$ & G & $T$ & $c$ & $G$ & $\mathrm{c}$ & $T$ & $\mathrm{c}$ & J1 \\
\hline 15 & $T$ & $G$ & A & $T$ & G & $T$ & A & $G$ & $T$ & $\mathrm{c}$ & $\mathrm{c}$ & $\mathrm{c}$ & $T$ & $\mathrm{c}$ & $\mathrm{c}$ & $G$ & A & 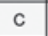 & $\mathrm{c}$ & $\mathrm{c}$ & $\mathrm{c}$ & G & $T$ & $\mathrm{c}$ & $G$ & $\mathrm{c}$ & $T$ & $\mathrm{c}$ & J1 \\
\hline 16 & $T$ & $G$ & A & $T$ & $G$ & $T$ & $A$ & $G$ & $T$ & c & c & $c$ & $T$ & $\mathrm{c}$ & $\mathrm{c}$ & $G$ & $A$ & $c$ & c & c & $c$ & $G$ & $T$ & c & $G$ & c & $T$ & c & J1 \\
\hline 17 & $T$ & G & A & $T$ & G & $T$ & A & G & $T$ & $\mathrm{c}$ & C & C & $T$ & C & $\mathrm{c}$ & G & $A$ & $\begin{array}{lc}\mathrm{c} \\
\end{array}$ & $\mathrm{C}$ & c & $\mathrm{c}$ & G & $\mathrm{T}$ & $\begin{array}{lc}\mathbf{c} \\
\end{array}$ & G & c & $T$ & $\mathrm{c}$ & J1 \\
\hline 18 & $T$ & G & A & $T$ & G & $T$ & A & G & $T$ & c & c & $c$ & $T$ & c & c & G & $A$ & $c$ & c & c & $c$ & G & $T$ & $c$ & G & $\mathrm{c}$ & $T$ & c & J1 \\
\hline 19 & $T$ & $G$ & A & $T$ & G & $T$ & A & G & $T$ & c & C & c & $T$ & $\mathrm{c}$ & $\mathrm{c}$ & $G$ & A & $c$ & $\mathrm{C}$ & c & $\mathrm{c}$ & G & $T$ & $\mathrm{c}$ & $G$ & $\mathrm{c}$ & $T$ & $\mathrm{c}$ & $\mathrm{J1}$ \\
\hline 20 & $T$ & 0 & A & $T$ & $G$ & $\mathrm{~T}$ & $A$ & 0 & $T$ & c & C & c & $T$ & c & c & $G$ & $A$ & c & c & c & $\mathrm{c}$ & $G$ & $T$ & $\mathrm{c}$ & $G$ & c & $T$ & c & J1 \\
\hline 21 & $T$ & G & A & $T$ & G & $\mathrm{T}$ & A & G & $T$ & $\mathrm{c}$ & $\mathrm{C}$ & $\mathrm{C}$ & $T$ & $\mathrm{C}$ & $\mathrm{c}$ & G & $A$ & $\mathrm{c}$ & $\mathrm{C}$ & C & $\mathrm{c}$ & G & $T$ & $\begin{array}{ll}\mathrm{C} \\
\end{array}$ & G & $\mathrm{c}$ & $T$ & $\mathrm{c}$ & \begin{tabular}{|l|} 
J1 \\
\end{tabular} \\
\hline 22 & $T$ & G & A & $T$ & G & $T$ & A & G & $T$ & c & c & c & $T$ & c & c & $G$ & A & c & c & c & c & G & $T$ & c & $G$ & c & $T$ & c & J1 \\
\hline 23 & $T$ & $G$ & A & $T$ & $G$ & $T$ & A & $G$ & $T$ & c & C & C & $T$ & $\mathrm{C}$ & c & $G$ & $A$ & $c$ & c & C & $\mathrm{c}$ & $G$ & $T$ & $\mathrm{c}$ & $G$ & c & $T$ & c & J1 \\
\hline 24 & $T$ & $G$ & A & $T$ & $G$ & $T$ & A & $G$ & $T$ & $\mathrm{C}$ & $\mathrm{C}$ & $\mathrm{c}$ & $T$ & $\mathrm{c}$ & $\mathrm{c}$ & $G$ & $A$ & $\mathrm{C}$ & $\mathrm{c}$ & $\mathrm{c}$ & $\mathrm{c}$ & G & $T$ & $\mathrm{c}$ & $G$ & $\mathrm{c}$ & $T$ & $\mathrm{C}$ & J1 \\
\hline 25 & $T$ & G & A & $T$ & G & $T$ & A & G & $T$ & c & c & c & $T$ & c & c & G & A & $c$ & c & c & $\mathrm{c}$ & $G$ & $T$ & c & $\mathrm{G}$ & c & $T$ & c & J1 \\
\hline 26 & $T$ & G & A & $T$ & $G$ & $T$ & A & G & $T$ & c & c & c & $T$ & c & c & $G$ & $A$ & c & c & c & c & $G$ & $T$ & c & $G$ & c & $T$ & c & \begin{tabular}{|l} 
J1 \\
\end{tabular} \\
\hline 27 & $T$ & $G$ & A & $T$ & $G$ & $T$ & A & 0 & $T$ & $\mathrm{c}$ & $\mathrm{c}$ & $c$ & $T$ & $\mathrm{C}$ & $\mathrm{c}$ & $G$ & $A$ & $\mathrm{c}$ & $\mathrm{C}$ & c & $\mathrm{c}$ & $G$ & $T$ & c & $G$ & $\mathrm{c}$ & $T$ & $\mathrm{c}$ & J1 \\
\hline 28 & $\mathbf{T}$ & G & A & $T$ & G & $\mathrm{T}$ & A & G & $T$ & c & c & c & $T$ & $\mathrm{C}$ & $\mathrm{c}$ & G & $A$ & c & c & c & $\mathrm{c}$ & G & $\mathrm{T}$ & $\mathrm{c}$ & G & c & $T$ & $\mathrm{c}$ & J1 \\
\hline 29 & $T$ & G & A & $T$ & G & $T$ & A & $G$ & $T$ & c & c & c & $T$ & c & c & G & A & $c$ & c & c & c & $G$ & $T$ & c & $G$ & c & $T$ & c & J1 \\
\hline 30 & $T$ & $G$ & A & $T$ & $G$ & $T$ & A & $G$ & $T$ & $\mathrm{c}$ & C & $\mathrm{c}$ & $T$ & $\mathrm{c}$ & $\mathrm{c}$ & $G$ & $A$ & $\mathrm{c}$ & $\mathrm{c}$ & $\mathrm{c}$ & $\mathrm{c}$ & G & $T$ & $\mathrm{c}$ & $G$ & $\mathrm{c}$ & $T$ & $\mathrm{c}$ & J1 \\
\hline 31 & $T$ & $G$ & A & $T$ & G & $T$ & A & $G$ & $T$ & C & C & c & $T$ & C & c & $G$ & $A$ & C & C & C & $\mathrm{c}$ & $G$ & $T$ & c & $G$ & c & $T$ & c & J1 \\
\hline 32 & $T$ & G & A & $T$ & G & $\mathrm{T}$ & A & G & $T$ & c & c & c & $T$ & $\mathrm{c}$ & c & G & A & c & c & c & $\mathrm{c}$ & G & $T$ & c & $G$ & c & $T$ & c & \begin{tabular}{|l} 
J1 \\
\end{tabular} \\
\hline 33 & $T$ & G & A & $T$ & $G$ & $T$ & A & G & $T$ & c & C & $c$ & $T$ & $\mathrm{c}$ & c & $G$ & $A$ & $c$ & C & c & $\mathrm{c}$ & $G$ & $T$ & $\mathrm{c}$ & $G$ & c & $T$ & c & J1 \\
\hline 34 & $T$ & $G$ & A & $T$ & $G$ & $T$ & $A$ & 0 & $T$ & c & c & c & $T$ & c & c & $G$ & $A$ & $c$ & c & c & $\mathrm{c}$ & $G$ & $T$ & c & $G$ & c & $T$ & c & \begin{tabular}{|l} 
J1 \\
\end{tabular} \\
\hline 35 & $T$ & G & A & $\mathrm{T}$ & G & $\mathrm{T}$ & $A$ & G & $T$ & c & C & c & $\mathrm{T}$ & C & c & G & A & $\mathrm{c}$ & C & c & c & G & $\mathrm{T}$ & c & $\mathrm{G}$ & c & $T$ & c & \begin{tabular}{|l} 
J1 \\
\end{tabular} \\
\hline 36 & $T$ & G & A & $T$ & G & $T$ & A & $G$ & $T$ & c & c & c & $T$ & $\mathrm{c}$ & $\mathrm{c}$ & G & A & c & c & c & $\mathrm{c}$ & $G$ & $T$ & $\mathrm{c}$ & $G$ & $\mathrm{c}$ & $T$ & c & J1 \\
\hline 37 & $T$ & 0 & A & $T$ & $G$ & $T$ & $A$ & $G$ & $T$ & c & C & c & $T$ & c & c & $G$ & $A$ & c & c & c & c & $G$ & $T$ & c & $G$ & c & $T$ & c & J1 \\
\hline 38 & $T$ & $G$ & A & $T$ & $G$ & $T$ & A & $G$ & $T$ & $\mathrm{c}$ & C & $c$ & $T$ & $\mathrm{c}$ & $\mathrm{c}$ & $G$ & $A$ & $c$ & $\mathrm{C}$ & c & $\mathrm{c}$ & $G$ & $T$ & $\mathrm{c}$ & $G$ & $\mathrm{c}$ & $T$ & $\mathrm{C}$ & J1 \\
\hline 39 & $T$ & G & A & $T$ & G & $\mathrm{T}$ & A & G & $T$ & c & c & c & $T$ & c & c & G & A & $c$ & c & c & $\mathrm{c}$ & $G$ & $T$ & c & G & c & $T$ & c & J1 \\
\hline 40 & $T$ & G & A & $T$ & $G$ & $T$ & A & G & $T$ & c & c & C & $T$ & c & c & $G$ & $A$ & c & c & c & c & $G$ & $T$ & c & $G$ & c & $T$ & c & J1 \\
\hline 41 & $T$ & 0 & A & $T$ & $G$ & $T$ & A & 0 & $T$ & c & c & c & $T$ & c & c & $G$ & A & $\mathrm{c}$ & c & c & c & G & $T$ & c & $G$ & c & $T$ & c & $\mathrm{J1}$ \\
\hline 42 & $\mathbf{T}$ & G & A & $T$ & G & $\mathrm{T}$ & A & G & $T$ & c & C & C & $T$ & C & c & G & $A$ & $\mathrm{c}$ & C & c & $\mathrm{c}$ & G & $\mathrm{T}$ & c & G & c & $T$ & c & J1 \\
\hline
\end{tabular}

Figure 2. (A) Genomic variations on open reading frames (ORFs) 1, 21 and 54 of 42 varicella-zoster virus (VZV) isolates collected from Yunnan province of Southwestern China. The sequence of the Dumas, MLS, vOka and pOka VZV strains referred to the published data. The sequence positions referred to the sequence of Dumas VZV strain (genebank accession no. NC_001348). Grey cells represents genotype C markers. Light grey cells represents genotype J markers. Dark grey cells represent various genetic variations (or mutations).

Fig. 2A. The VZV isolates could be grouped into genotype J (41/42) or M2 (1/42) according to the genotyping scheme of Loparev et al (13), as shown in Fig. 2B.

\section{Discussion}

VZV is highly infectious for those with no VZV infection history, and becomes dormant in the sensory ganglia following the first infection. The genome of VZV is highly conserved compared to other pathological viruses, such as human papilloma virus. This may be due to the low reproduction of VZV in the infected hosts. This feature limits the frequency of the introduction of new mutations in VZV genes. As the advancement of VZV molecular epidemiology occurs, numerous genomic variations have been identified in VZV strains. Based on these genomic variations, the VZV strains have been classified into different genotypes. The VZV genotypes in China are rarely studied. The present study made a preliminary study on the genotypes of VZV isolates collected from Yunnan province of Southwestern China. To the best of 


\begin{tabular}{|c|c|c|c|c|c|c|c|c|}
\hline Gene & & & & DRF2 & & & & \\
\hline $\begin{array}{l}\text { Nucleotide } \\
\text { Position }\end{array}$ & $\begin{array}{l}\text { W } \\
\text { ֻ }\end{array}$ & 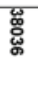 & $\begin{array}{l}\text { 䍐 } \\
\text { 品 }\end{array}$ & 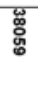 & $\begin{array}{l}\text { 吅 } \\
\text { 茞 }\end{array}$ & $\begin{array}{l}\mathscr{\Phi} \\
\stackrel{\Xi}{\sharp}\end{array}$ & 芯 & 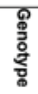 \\
\hline Dumas & A & $T$ & $T$ & $\mathrm{C}$ & A & $G$ & A & E \\
\hline MLS [17] & A & $T$ & $T$ & $\mathrm{C}$ & c & G & A & M1 \\
\hline vOka [17] & G & c & $c$ & $\mathrm{C}$ & $c$ & $A$ & A & $J$ \\
\hline poka [17] & G & $T$ & C & $\mathrm{C}$ & C & A & A & $\mathrm{J}$ \\
\hline 1 & $G$ & $T$ & c & $\mathrm{C}$ & c & A & A & $\mathrm{J}$ \\
\hline 2 & $G$ & $T$ & c & $\mathrm{C}$ & c & A & A & $\mathrm{J}$ \\
\hline 3 & G & $T$ & c & $\mathrm{C}$ & $c$ & A & A & $\mathrm{J}$ \\
\hline 4 & G & $T$ & C & $\mathrm{C}$ & C & A & A & $J$ \\
\hline 5 & G & $T$ & c & $\mathrm{C}$ & c & A & A & $J$ \\
\hline 6 & G & $T$ & c & $\mathrm{c}$ & c & A & A & $J$ \\
\hline 7 & G & $T$ & c & $\mathrm{C}$ & c & A & A & $\mathrm{J}$ \\
\hline 8 & G & $T$ & c & $\mathrm{c}$ & $c$ & A & $A$ & $\mathrm{~J}$ \\
\hline 9 & G & $T$ & c & $\mathrm{C}$ & c & $A$ & A & $\mathrm{J}$ \\
\hline 10 & A & $T$ & c & $\mathrm{C}$ & c & $A$ & A & M2 \\
\hline 11 & $G$ & $T$ & c & $\mathrm{C}$ & c & A & A & $J$ \\
\hline 12 & $G$ & $T$ & c & $\mathrm{c}$ & c & $A$ & $A$ & $J$ \\
\hline 13 & G & $T$ & c & $\mathrm{C}$ & c & A & A & $J$ \\
\hline 14 & G & $T$ & c & $\mathrm{c}$ & $c$ & $A$ & A & $J$ \\
\hline 15 & $G$ & $T$ & c & $\mathrm{C}$ & c & A & $A$ & $\mathbf{J}$ \\
\hline 16 & $G$ & $T$ & c & $\mathrm{C}$ & $c$ & A & $A$ & $\mathrm{~J}$ \\
\hline 17 & G & $T$ & C & $\mathrm{C}$ & c & A & $A$ & $\mathbf{J}$ \\
\hline 18 & G & $T$ & $\mathrm{C}$ & $\mathrm{C}$ & $\mathrm{c}$ & A & A & $\mathrm{J}$ \\
\hline 19 & G & $T$ & C & $\mathrm{C}$ & c & A & A & $\mathbf{J}$ \\
\hline 20 & G & $T$ & $\mathrm{C}$ & $\mathrm{c}$ & $\mathrm{c}$ & $A$ & $A$ & $\mathrm{~J}$ \\
\hline 21 & G & $T$ & $\mathrm{C}$ & $\mathrm{C}$ & $\mathrm{C}$ & $A$ & $A$ & $\mathrm{~J}$ \\
\hline 22 & G & $T$ & $\mathrm{C}$ & $\mathrm{C}$ & $\mathrm{c}$ & $A$ & $A$ & $\mathrm{~J}$ \\
\hline 23 & $G$ & $T$ & $\mathrm{c}$ & $\mathrm{c}$ & $c$ & $A$ & $A$ & $\mathbf{J}$ \\
\hline 24 & G & $T$ & $\mathrm{C}$ & $\mathrm{C}$ & C & $A$ & A & $\mathbf{J}$ \\
\hline 25 & G & $T$ & $\mathrm{c}$ & $\mathrm{c}$ & $c$ & $A$ & A & $\mathrm{J}$ \\
\hline 26 & G & $T$ & $\mathrm{C}$ & $\mathrm{c}$ & $\mathrm{c}$ & $A$ & A & $\mathrm{J}$ \\
\hline 27 & G & $T$ & $\mathrm{c}$ & $\mathrm{c}$ & c & $A$ & $A$ & $\mathbf{J}$ \\
\hline 28 & G & $T$ & $\mathrm{C}$ & $\mathrm{c}$ & c & $A$ & $A$ & $\mathrm{~J}$ \\
\hline 29 & G & $T$ & $\mathrm{c}$ & $\mathrm{c}$ & c & $A$ & A & $\mathbf{J}$ \\
\hline 30 & G & $T$ & $\mathrm{C}$ & $\mathrm{C}$ & $\mathrm{C}$ & $A$ & A & $\mathbf{J}$ \\
\hline 31 & 0 & $T$ & $\mathrm{c}$ & $\mathrm{c}$ & c & $A$ & $A$ & $\mathrm{~J}$ \\
\hline 32 & G & $T$ & $\mathrm{C}$ & $\mathrm{C}$ & C & $A$ & A & $\mathrm{J}$ \\
\hline 33 & $G$ & $T$ & $\mathrm{C}$ & $\mathrm{c}$ & $\mathrm{c}$ & A & A & $\mathrm{J}$ \\
\hline 34 & $G$ & $T$ & $\mathrm{C}$ & $\mathrm{C}$ & c & $A$ & $A$ & $\mathrm{~J}$ \\
\hline 35 & G & $\mathrm{T}$ & $\mathrm{C}$ & $\mathrm{C}$ & $\mathrm{c}$ & A & A & $\mathrm{J}$ \\
\hline 36 & G & $T$ & C & $\mathrm{C}$ & c & A & A & $\mathrm{J}$ \\
\hline 37 & G & $\mathrm{T}$ & $\mathrm{c}$ & $\mathrm{C}$ & $\mathrm{C}$ & A & A & $\mathrm{J}$ \\
\hline 38 & G & $T$ & $\mathrm{C}$ & $\mathrm{C}$ & C & A & A & $\mathrm{J}$ \\
\hline 39 & G & $T$ & C & $\mathrm{c}$ & $\mathrm{C}$ & A & A & $\mathbf{J}$ \\
\hline 40 & G & $T$ & $\mathrm{C}$ & $\mathrm{C}$ & $\mathrm{c}$ & A & A & $\mathrm{J}$ \\
\hline 41 & G & $T$ & C & $\mathrm{C}$ & C & A & A & $\mathrm{J}$ \\
\hline 42 & G & $T$ & $\mathrm{C}$ & $\mathrm{C}$ & c & $A$ & $A$ & $\mathrm{~J}$ \\
\hline
\end{tabular}

Figure 2. Continued. (B) Genomic variations of open reading frame (ORF) 22 of 42 varicella-zoster virus (VZV) isolates collected from Yunnan province of Southwestern China. The sequence of the Dumas, MLS, vOka and pOka VZV strains referred to the published data. The sequence positions referred to the sequence of Dumas VZV strain (genebank accession no. NC_001348). Grey cells represents genotype C markers. Light grey cells represents genotype J markers. Dark grey cells represents various genetic variations (or mutations).

our knowledge, this is the first investigation on VZV genotype in this region of China.

The genotype of VZV strains is associated with climate. In temperate regions, the VZV strains are often $P s t \mathrm{I}^{+}$and $B g l \mathrm{I}$ negative $\left(B g l \mathrm{I}^{-}\right)$in ORF38 and ORF54, while the majority of VZV strains in tropical regions are $P s t \mathrm{I}^{+}$and $B g l \mathrm{I}^{+}(13,20-22)$. In the present study, the VZV isolates collected from the Yunnan province of Southwestern China were all $\mathrm{Pst} \mathrm{I}^{+}$and $\mathrm{Bg} / \mathrm{I}^{+}$in ORF38 and ORF54 (Fig. 1A and Table II). The present results are consistent with the findings that the VZV strains in the tropical regions are often $P s t \mathrm{I}^{+}$and $B g l \mathrm{I}^{-}$in ORF38 and ORF54, as the Yunnan province of China is located in the tropical regions.

ORF38 (PstI), ORF54 (BglI) and ORF62 (SmaI) in VZV genes are molecular genetic markers for the genotyping of VZV strains $(18,20,21)$. The VZV isolates in the present study were all $P s t \mathrm{I}^{+}, B g l \mathrm{I}^{+}$and $\mathrm{SmaI}^{+}$(Fig. 1 and Table II). The VZV isolates collected from Anhui city of China and the strain MLS (6) were $P s t \mathrm{I}^{+}, B g l \mathrm{I}^{+}$and $S m a \mathrm{I}^{-}$(wild-type VZV). The strain p-Oka was Pst $\mathrm{I}^{-}, B g l \mathrm{I}^{+}$and $S m a \mathrm{I}^{-}(17)$. The strain v-Oka was $P s t \mathrm{I}^{-}, B g l \mathrm{I}^{+}$and $S m a \mathrm{I}^{+}(17)$. The results indicated that the VZV isolates collected from the Yunnan province of Southwestern China were different from that of Anhui city of middle eastern China. The results supported the conclusion that the genotype of VZV strains may vary in different regions of China. Further studies are required to find out the VZV distributions in China.

The R5 variable region in the VZV genes has been shown to be geographically related $(10,23)$. The type of R5A (359 bp) is mainly found in Europe and North America. The type of $\mathrm{R} 5 \mathrm{~B}$ (471 bp) is a major type in Japan. In the present study, R5A and R5B were observed in the collected VZV isolates (Fig. 1). The genotype frequency of R5A and R5B are nearly identical in these samples. The results are consistent with the findings by Liu et al (17). It is possible that either R5A or R5B is equally distributed in Chinese VZV strains.

Until now, there was no gold standard VZV genotyping scheme, although several genotyping schemes have been proposed in recent years. The genetic variations were identified in VZV ORFs 1, 21, 22, 50 and $54(17,19,24,25)$. By referring to the SNPs in VZV ORFs 1,21, 50 and 54, the VZV strains could be grouped into 4 genotypes: A, B, C and J. By referring to the VZV ORF22, 7 VZV genotypes are identified, which were E1, E2, J, M1, M2, M3 and M4. The VZV strains of genotype $\mathbf{J}$ are most common in Asia, particular in Japan. In the present study, the above genotyping scheme was used to analyze the VZV samples collected from the Yunnan province of Southwestern China (Fig. 2). The collected VZV isolates are mainly genotype $\mathrm{J}$ or $\mathrm{J} 1$, which is a major genotype in Asia. The results are consistent with the finding by Liu et al (17), who collected the VZV isolates from Anhui city of middle eastern China, which were genotyped as J or J1. In addition to the previously reported genetic variations in the VZV genes, no new genetic variations were identified in the collected VZV isolates by sequencing the ORFs 1, 21, 22 and 54 in VZV genes. The results indicated that the VZV strains in Yunnan province may be highly conserved, as several reports have shown new genetic variations in these sequenced fragments. Of note, one sample in the collected isolates was classified as genotype A1 or M2. This may be due to the population migrations.

In conclusion, a preliminary study was performed on VZV genotypes in the Yunnan province of Southwestern China. The results of the present study will aid in the understanding of the genetics of VZV in China. The limitation of the study is that the sample size is small, although it is larger than the previous 
studies in China. Further studies with a larger sample size are required to understand the VZV genotype distributions in this region and China.

\section{Acknowledgements}

The present study was supported by the Education Commission of Yunnan Province (grant no. 2014Z036) and the Kunming University of Science and Technology (grant no. KKZ3201360025). The authors would like to thank Dr Ping Cao for collecting the VZV samples, and the patients who participated.

\section{References}

1. Nagel MA, Cohrs RJ, Mahalingam R, et al: The varicella zoster virus vasculopathies: Clinical, $\mathrm{CSF}$, imaging and virologic features. Neurology 70: 853-860, 2008.

2. Elkind MS: The varicella zoster virus vasculopathies: Clinical, CSF, imaging, and virologic features. Neurology 72: 1028-1030; author reply 129-130, 2009

3. Nagel MA and Gilden DH: The protean neurologic manifestations of varicella-zoster virus infection. Cleve Clin J Med 74 489-494, 496, 498, 499 passim, 2007.

4. Becerra JC, Sieber R, Martinetti G, et al: Infection of the central nervous system caused by varicella zoster virus reactivation: A retrospective case series study. Int J Infect Dis 17: e529-e534, 2013.

5. Peters GA, Tyler SD, Grose C, et al: A full-genome phylogenetic analysis of varicella-zoster virus reveals a novel origin of replication-based genotyping scheme and evidence of recombination between major circulating clades. J Virol 80: 9850-9860, 2006.

6. Barrett-Muir W, Scott FT, Aaby P, et al: Genetic variation of varicella-zoster virus: Evidence for geographical separation of strains. J Med Virol 70 (Suppl 1): S42-S47, 2003.

7. Carr MJ, McCormack GP, and Crowley B: Genetic variation in clinical varicella-zoster virus isolates collected in Ireland between 2002 and 2003. J Med Virol 73: 131-136, 2004.

8. Muir WB, Nichols R and Breuer J: Phylogenetic analysis of varicella-zoster virus: Evidence of intercontinental spread of genotypes and recombination. J Virol 76: 1971-1979, 2002.

9. Faga B, Maury W, Bruckner DA, et al: Identification and mapping of single nucleotide polymorphisms in the varicella-zoster virus genome. Virology 280: 1-6, 2001.

10. Hawrami K and Breuer J: Analysis of United Kingdom wild-type strains of varicella-zoster virus: Differentiation from the Oka vaccine strain. J Med Virol 53: 60-62, 1997.

11. Hawrami K, Harper D and Breuer J: Typing of varicella zoster virus by amplification of DNA polymorphisms. J Virol Methods 57: 169-174, 1996.
12. Loparev VN, Argaw T, Krause PR, et al: Improved identification and differentiation of varicella-zoster virus (VZV) wild-type strains and an attenuated varicella vaccine strain using a VZV open reading frame 62-based PCR. J Clin Microbiol 38: 3156-3160, 2000.

13. Loparev VN, Gonzalez A, Deleon-Carnes M, et al: Global identification of three major genotypes of varicella-zoster virus: Longitudinal clustering and strategies for genotyping. J Virol 78: 8349-8358, 2004.

14. Norberg P, Liljeqvist JA, Bergstrom T, et al: Complete-genome phylogenetic approach to varicella-zoster virus evolution: Genetic divergence and evidence for recombination. J Virol 80: 9569-9576, 2006.

15. Sergeev N, Rubtcova E, Chizikov V, et al: New mosaic subgenotype of varicella-zoster virus in the USA: VZV detection and genotyping by oligonucleotide-microarray. J Virol Methods 136: 8-16, 2006.

16. Chow VT, Tipples GA and Grose C: Bioinformatics of varicella-zoster virus: Single nucleotide polymorphisms define clades and attenuated vaccine genotypes. Infect Genet Evol 18: 351-356, 2013.

17. Liu J, Wang M, GanL, et al: Genotyping of clinical varicella-zoster virus isolates collected in China. J Clin Microbiol 47: 1418-1423, 2009.

18. Jiang L, Gan L, Chen J, et al: Genetic analysis of clinical VZV isolates collected in China reveals a more homologous profile. Biomed Res Int 2013: 681234, 2013.

19. Weidmann M, Meyer-König U and Hufert FT: Rapid detection of herpes simplex virus and varicella-zoster virus infections by real-time PCR. J Clin Microbiol 41: 1565-1568, 2003.

20. LaRussa P, Steinberg S, Arvin A, et al: Polymerase chain reaction and restriction fragment length polymorphism analysis of varicella-zoster virus isolates from the United States and other parts of the world. J Infect Dis 178 (Suppl 1): S64-S66, 1998.

21. LaRussa P, Lungu O, Hardy I, et al: Restriction fragment length polymorphism of polymerase chain reaction products from vaccine and wild-type varicella-zoster virus isolates. J Virol 66: 1016-1020, 1992.

22. Quinlivan M, Gershon AA, Steinberg SP, et al: An evaluation of single nucleotide polymorphisms used to differentiate vaccine and wild type strains of varicella-zoster virus. J Med Virol 75: 174-180, 2005 .

23. Sauerbrei A, Eichhorn U, Gawellek S, et al: Molecular characterisation of varicella-zoster virus strains in Germany and differentiation from the Oka vaccine strain. J Med Virol 71: 313-319, 2003.

24. Quinlivan M, Hawrami K, Barrett-Muir W, et al: The molecular epidemiology of varicella-zoster virus: Evidence for geographic segregation. J Infect Dis 186: 888-894, 2002.

25. Bostikova V, Bostik P, Chlibek R, et al: Molecular epidemiology of varicella zoster virus. Epidemiol Mikrobiol Imunol 59: 21-24, 2010. 